\title{
BURNAUT-SYNDROM'S PREVENTION SCREENING
}

\author{
J. Gospodinova, K. Gospodinov, V. Goncchev, A. Halil \\ Home for Medical and Social Care for Children - Kardzhali, Bulgaria \\ Universety Assen Zlatarov, Burgas, Bulgaria
}

\begin{abstract}
In recent years, the evidence has shown that some professions are more vulnerable to mental exhaustion syndrome. The environment, characterized by the intensity of work with other people where the professionals are giving, and the counterpart is receiving help is very risky. The "burn-out" syndrome can lead to a number of consequences, reflecting extremely negatively on the professional development of the person. In the article we present to you the results obtained from our staff survey in HMSCC in Kardzhali through a convenient administration and accurate test method, enabling quick and economical detection of the instantly affective state and taking into account variations in the emotional state of the investigated person. We believe that the trend of a very strong emotional experience at the end of the working day, against the backdrop of moderate indicators taking into account most of the people surveyed, may be a signal for taking other person-specific measures and clarifying the situation - with a preventive purpose. We believe that the test is a cost-effective tool that can be applied in practice to other institutions as it is a reliable tool for the prevention of the bledout syndrome.
\end{abstract}

Key words: mental exhaustion syndrome, "burnout" syndrome

\section{INTRODUCTION}

Emotions have a practical impact on all aspects of people's lives and their functioning. They are in direct dependence on the peculiarities and significance of some external or internal irritant. Their magnitude and durability is also determined by the temperament of the individual, his life experience, the environment in which he is, the presence of fatigue, and other factors. The term "stress" has gained popularity since the end of World War II in the United States, when it is clear that the frontline situation can lead to pronounced psychological and physiological changes (1). In psychology, the term stress is used in the sense of an external factor causing some pathological change. Selley's name is the beginning of the research of stress. Stress is a conscious and real mismatch between the requirements of the surrounding environment and the individual opportunities for meeting them . There are different types and degrees of emotional responses among different individuals. Interestingly, agerelated stress factors, development crises. In each stage of the individual's age development, there are stressors that can condition the deployment of diverse conditions, often leading to disease breakdowns. Personality stress factors are another heterogeneous group. They are essential mainly in individuals with marked sensitivity and easy wounds, causing disorganization of their adaptive abilities and falling into unpredictable and difficult to manage emotional stressful states. Physiological changes and performance indicators are important indicators of stress. As a result of the stress, functional changes occur in the body and have an organizing character for enhancing the functional capacity. Over the last few years, evidence has shown that many professions are vulnerable to poverty, but in some of them there is a greater likelihood of it occurring (2).

As such, the following are defined (Figure 1).

The main risk factors for occupational stress can be divided in general in 3 groups (Figure 2).

He environment, characterized by the intensity of work with other people where the professionals are giving, and the counterpart is receiving help is very risky. The "burn-out" syndrome has three aspects:

- Developing a strong sense of emotional exhaustion - Developing a strong sense of emotional exhaustion - leads to the absence of any kind of emotional reaction, disinterest and apathy

- Disorganization - a tendency to develop negative attitudes towards those whose life and 
health the professional cares for and responds to, and the sense that he has become more insensitive than a person
- Reduced ability to work - tendency for negative self-esteem, sense of personal incompetence.

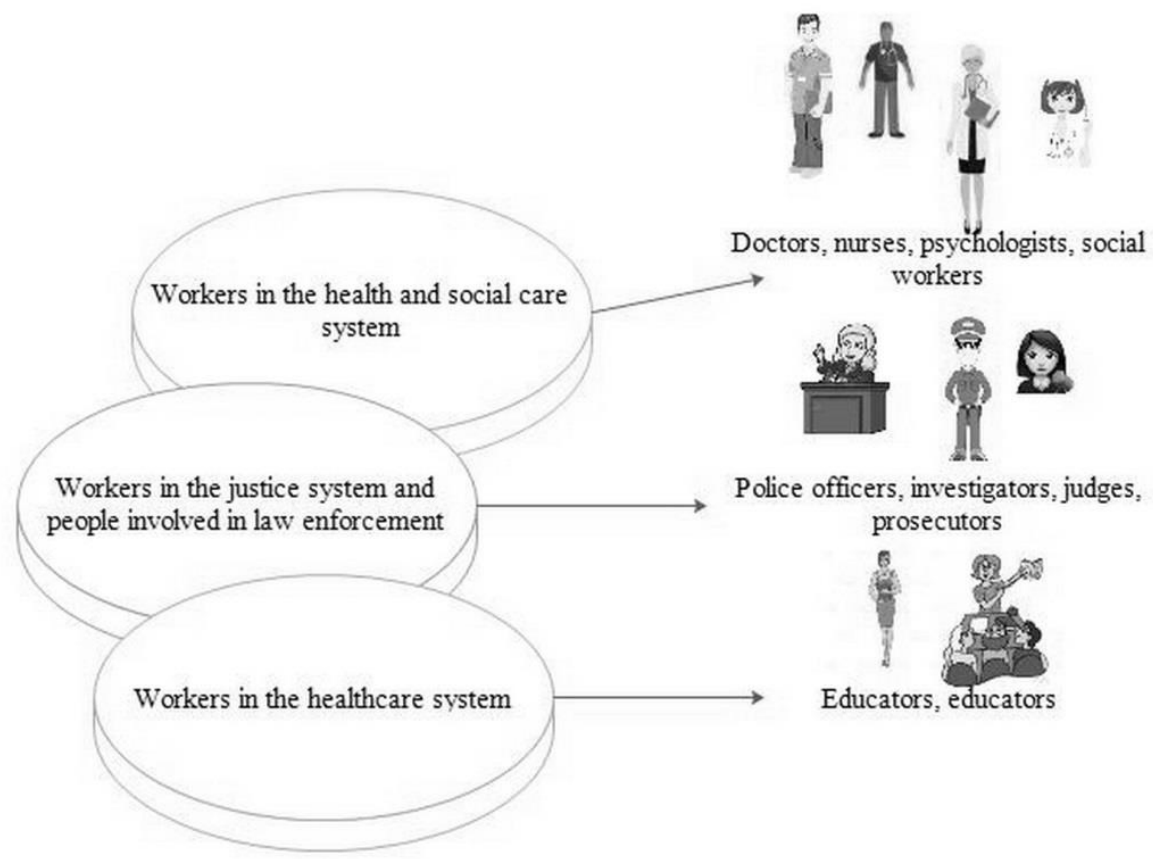

Figure 1. Risky professions

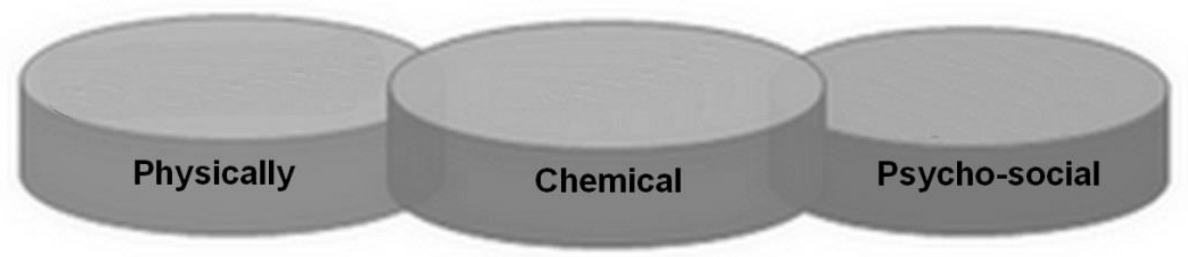

Figure 2. Key Risk Factors

If this process is left unattended for a long time, its interruption becomes very difficult. Burnout syndrome can be understood as the last phase of unsuccessful attempts by the person to deal with negative conditions of the professional environment, the result of unbridled social stress. Burnout syndrome is seen as a complex of symptoms and behaviors (3):

- Emotional exhaustion

- Helplessness

- Negative attitudes and feelings towards people who are dependent

- Conflicts with colleagues, depersonalisation

- Job dissatisfaction

- Trend of negative self-esteem in terms of selfemployment

- Anxiety

- Depression

- Hostility

- Non-sensitivity, negativism, etc.

- Reduced productivity

- Suicidal thoughts

- Paranoia
- Apathy

- Frustration

\section{PURPOSE, TASKS, HYPOTHESIS}

To establish a way to prevent Mental Healing Syndrome in our institution, we introduced a screening system that allows a quick and economical detection of the instantaneous affective state of the staff directly responsible for care for the children growing up in a home for medical and social care for children - Kardzhali at the beginning and the end of the working day, and for taking into account the variations in the emotional state of the investigated person. Our goal was to find out whether there is a trend towards a significant change in the affective states of those directly involved in caring for children growing up in an institution during the work process due to the specifics of working in homes for medical and social care for children. The tasks we set up were as follows:

- to examine the affective state of part of the staff at a home for medical and social care for 
children - Kardzhali, before and after the end of the working process;

- to analyze variations in the emotional state;

- to determine the degree of change in the positive and negative values obtained on the scales and dimensions;

- to identify the peculiarities of the individual differences in the results or the presence of similar tendencies in the affective states of the staff.

The hypothesis that emerged from our research was as follows: Studies investigating the staff's protective responses to institution-based homes for medical and social care for children prove the existence of the phenomenon of protection from attachment that causes deep discomfort. We assume that the early discovery of signs that give rise to persistent or immediate doubts about the professionalism of a staff member directly
GOSPODINOVA J., et al. involved in childcare will help prevent emotional disorders and also create conditions that contribute to violation of children's rights. We believe that the trend of a very strong emotional experience at the end of the working day, against the backdrop of moderate indicators reported by the majority of people surveyed, may be a signal for taking other person-specific measures and clarifying the situation - with a preventive purpose. We believe that staff in homes for medical and social care are more vulnerable to occupational burnout syndrome, which is likely to be apparent in the analysis of the results.

\section{PEOPLE STUDIED}

The experimental group of our study included a total of 32 female females aged 24-62 (Diagram1)

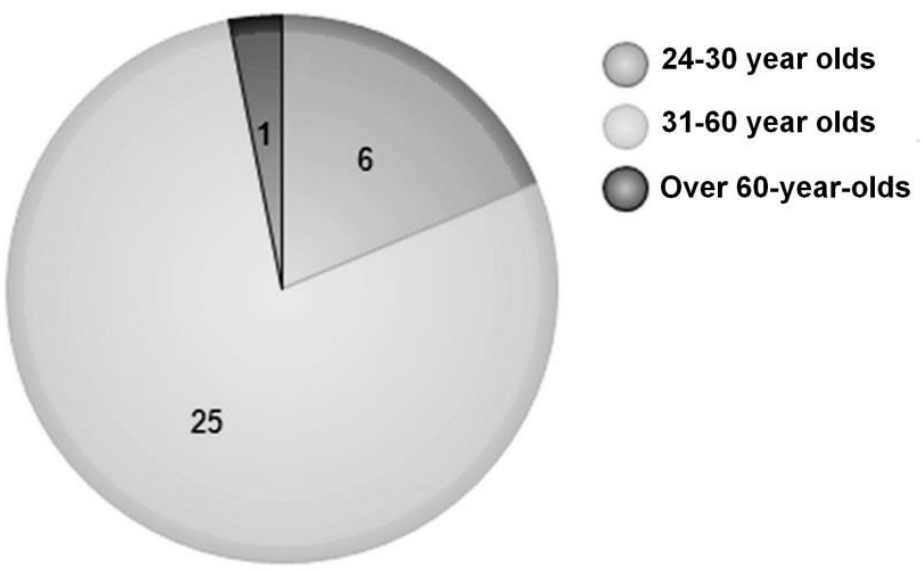

Diagram 1. Age distribution of surveyed individuals

who were directly involved in the care of children medical-and 7 are psychological-pedagogical growing up in a home for medical and social care for children in Kardzhali. Of these: 25 are

staffs (Diagram 2).

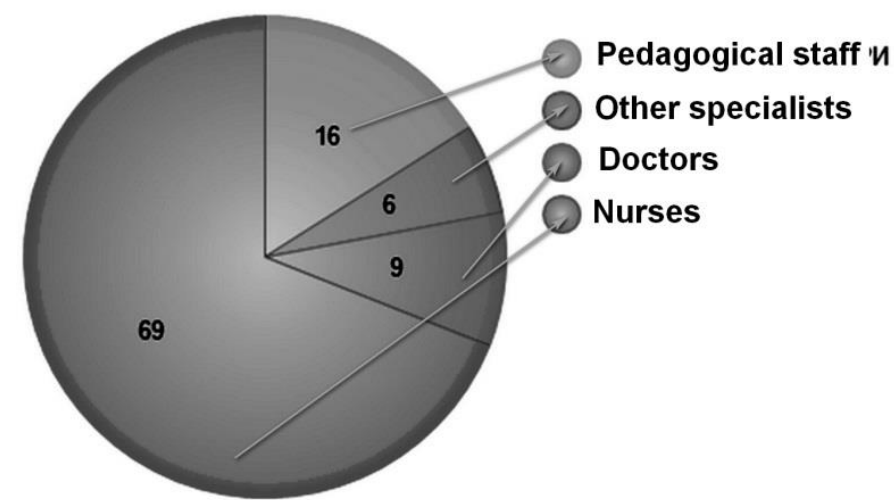

Diagram 2. Percentage distribution by occupations in total for all persons sur

\section{METHOD}

In order to establish the momentary affective status of the staff directly responsible for the care of children growing up at HOME for MEDICALSOCIAL CHILDREN CARE-Kardzhali, we used

a test method developed specifically for this purpose (4). Angel Velichkov, based on the work 
of Schimmack and Grob, develops a method designed to quickly and economically assess the emotional state. The method allows to assess the instantaneous affective state in order to account for its influence on the behavior or to trace its change under the influence of external factors. It is an economical tool that is quickly filled in and allows for the recording of variations in the affective state of the investigated person (Table 1 and Table 2). The method contains the following instructions: "Please appreciate what your selfesteem is at this time. From each pair of words describing the mood, choose the one that matches the tone you are experiencing now as you complete the test. To evaluate the power of the experience, use one of the following evaluations:
GOSPODINOVA J., et al.

0 - I do not feel either (neutral)

1 - I feel this feeling weak

2 - I feel this feeling moderate

3 - Feeling this feeling strong

4 - I feel this feeling very loud ".

The first (Table 1) two tables refer to the "activation-alertness" dimension, the next two (Table 2) are referred to as "activation-intensity" and the latter two refer to the assessment of the emotional tone. The estimate for each dimension is obtained as the sum of the readings on the respective scales. To distinguish the direction of the emotional state of the evaluations relative to the negative pole are attributed negative values.

Table 1. Assessment of the power of the experience

\begin{tabular}{|lllllllllllll|}
\hline \hline tension & 4 & 3 & 2 & 1 & 0 & 1 & 2 & 3 & 4 & relaxation \\
\hline nervousness & 4 & 3 & 2 & 1 & 0 & 1 & 2 & 3 & 4 & calmness \\
\hline cheerfulness & 4 & 3 & 2 & 1 & 0 & 1 & 2 & 3 & 4 & cheerfulness \\
\hline fresh mood & 4 & 3 & 2 & 1 & 0 & 1 & 2 & 3 & 4 & fatigue \\
\hline nice feeling & 4 & 3 & 2 & 1 & 0 & 1 & 2 & 3 & 4 & unpleasant feeling \\
\hline joy & 4 & 3 & 2 & 1 & 0 & 1 & 2 & 3 & 4 & sadness \\
\hline
\end{tabular}

Table 2. Drawings on the different di

\begin{tabular}{|c|c|c|}
\hline dimensions & & cales \\
\hline \multirow[t]{2}{*}{ Activation - cheerfulness } & tension & relaxation \\
\hline & nervousness & calmness \\
\hline \multirow[t]{2}{*}{ Activation - tension } & cheerfulness & indolence \\
\hline & fresh mood & fatigue \\
\hline \multirow[t]{2}{*}{ Emotional tone } & pleasant feeling & unpleasant feeling \\
\hline & joy & sadness \\
\hline
\end{tabular}

\section{RESULTS}

The results of the analysis of the response to the test after the survey show (Diagrams 3, 4, 5, 6, 7, $\mathbf{8 , 9 )}$ that there is a tendency for changes in the affective states during the work process for persons working in the homes for medical and social care for children. This change is related to the transition from positive to negative emotional experiences (Diagrams 3, 4, 5, 6, 7, 8, 9).

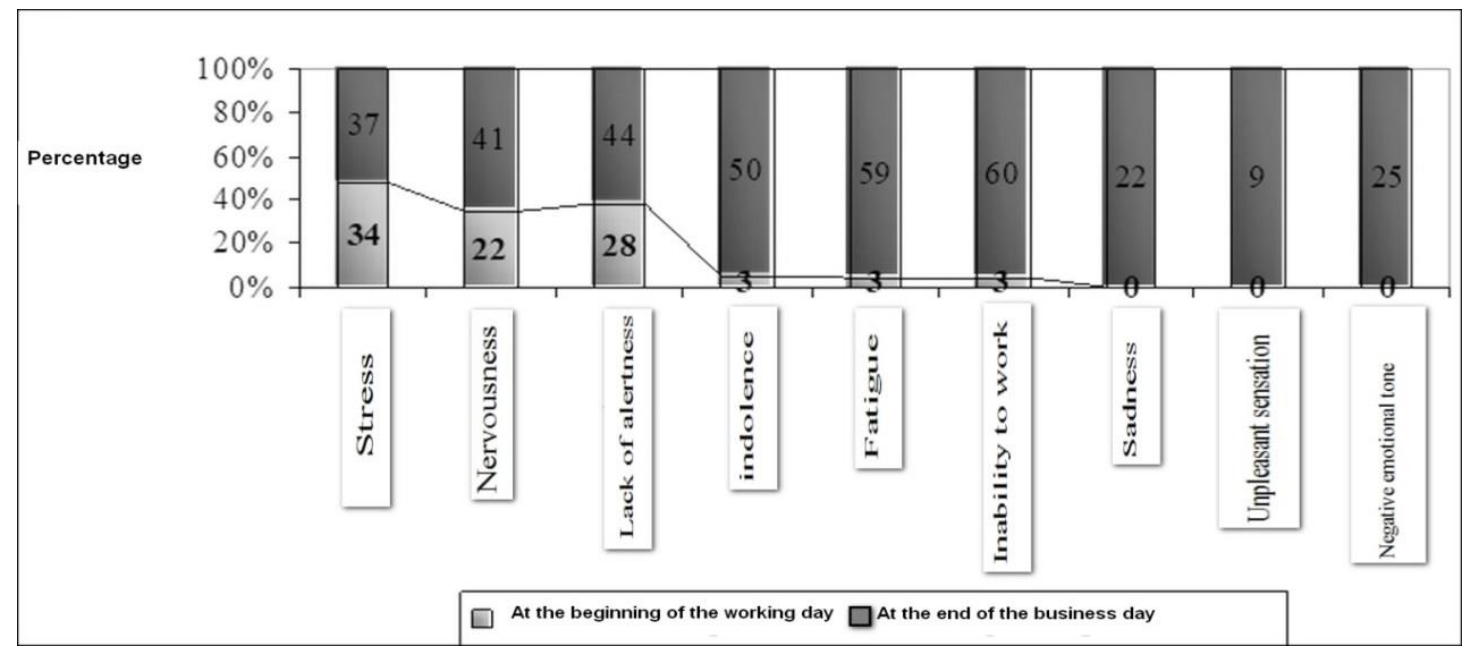

Diagram 3. Negative answers in the beginning and at the end of the working day 


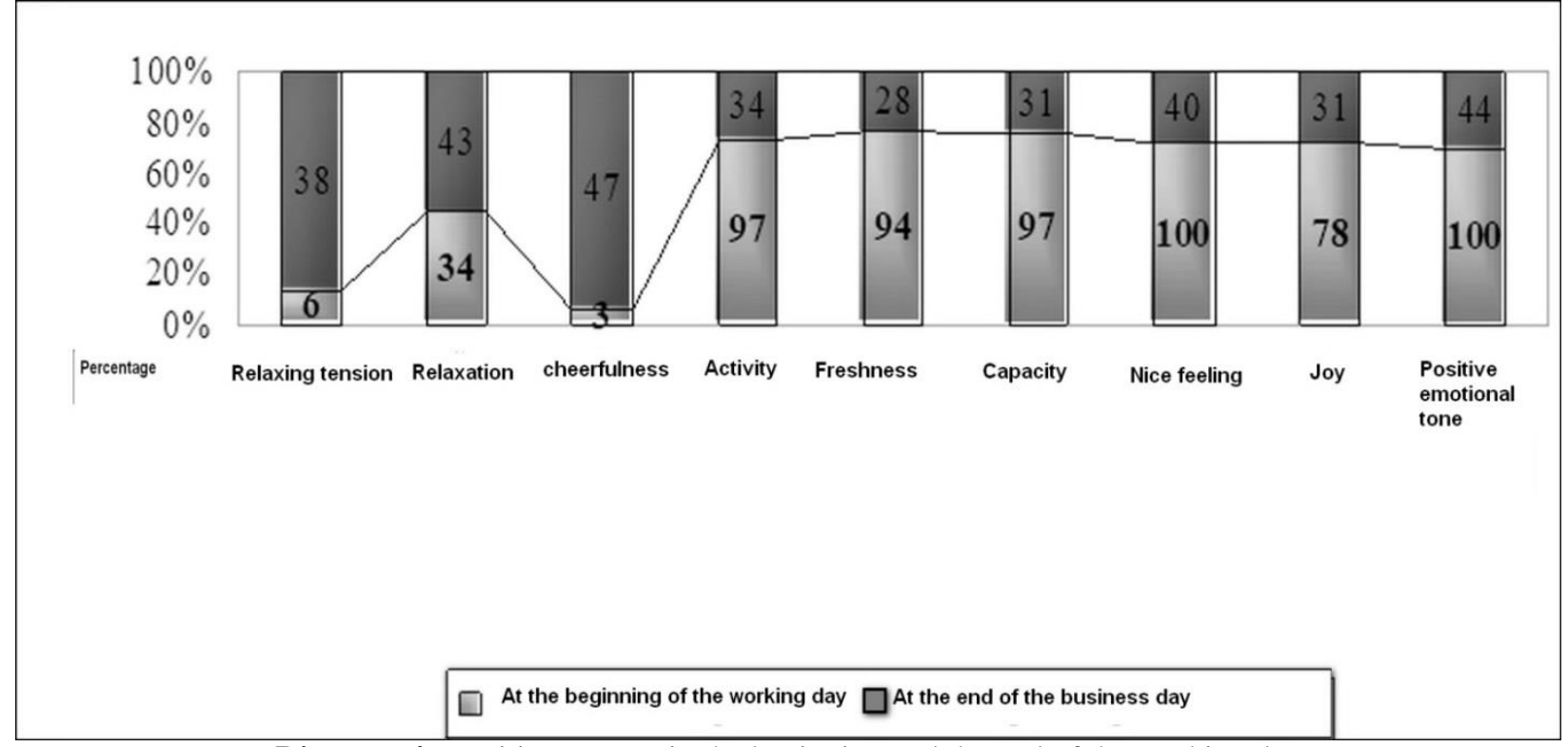

Diagram 4. Positive answes in the beginning and the end of the working day

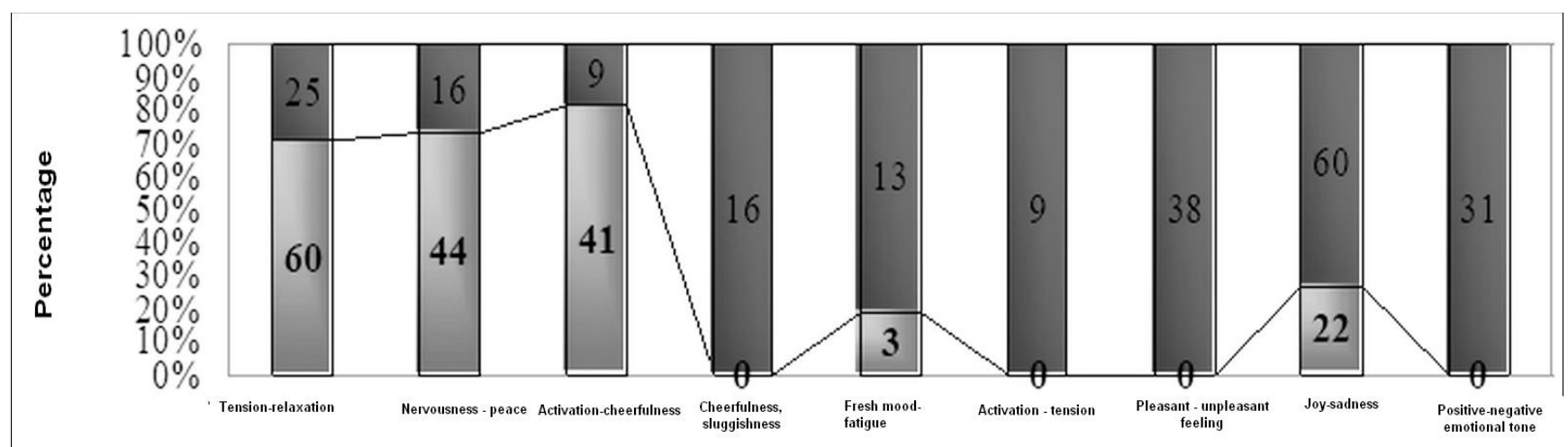

At the beginning of the working day $\square$ At the end of the business day

Diagram 5. Neutral answers in the beginning and the end of the working day

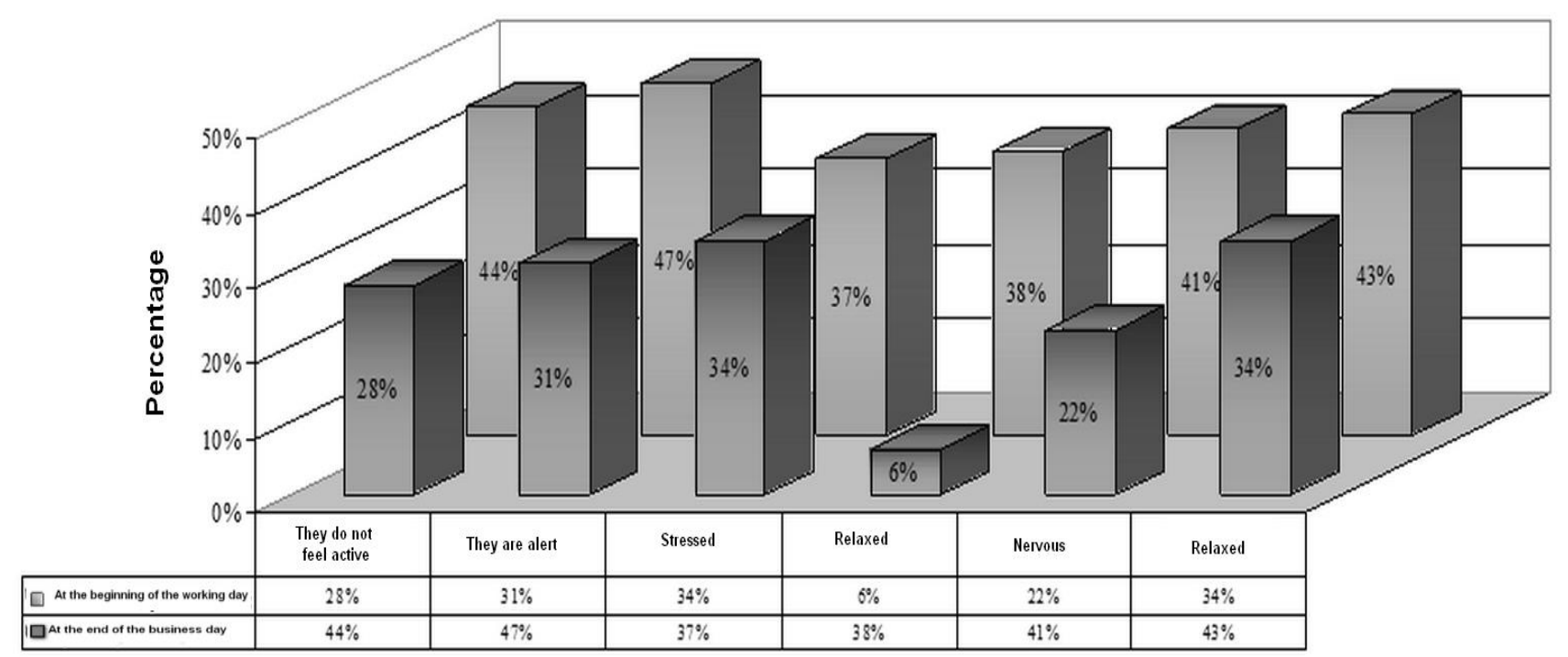

Diagram 6. Results on dimension activation bodrost

Trakia Journal of Sciences, Vol. 16, Suppl. 1, 2018 


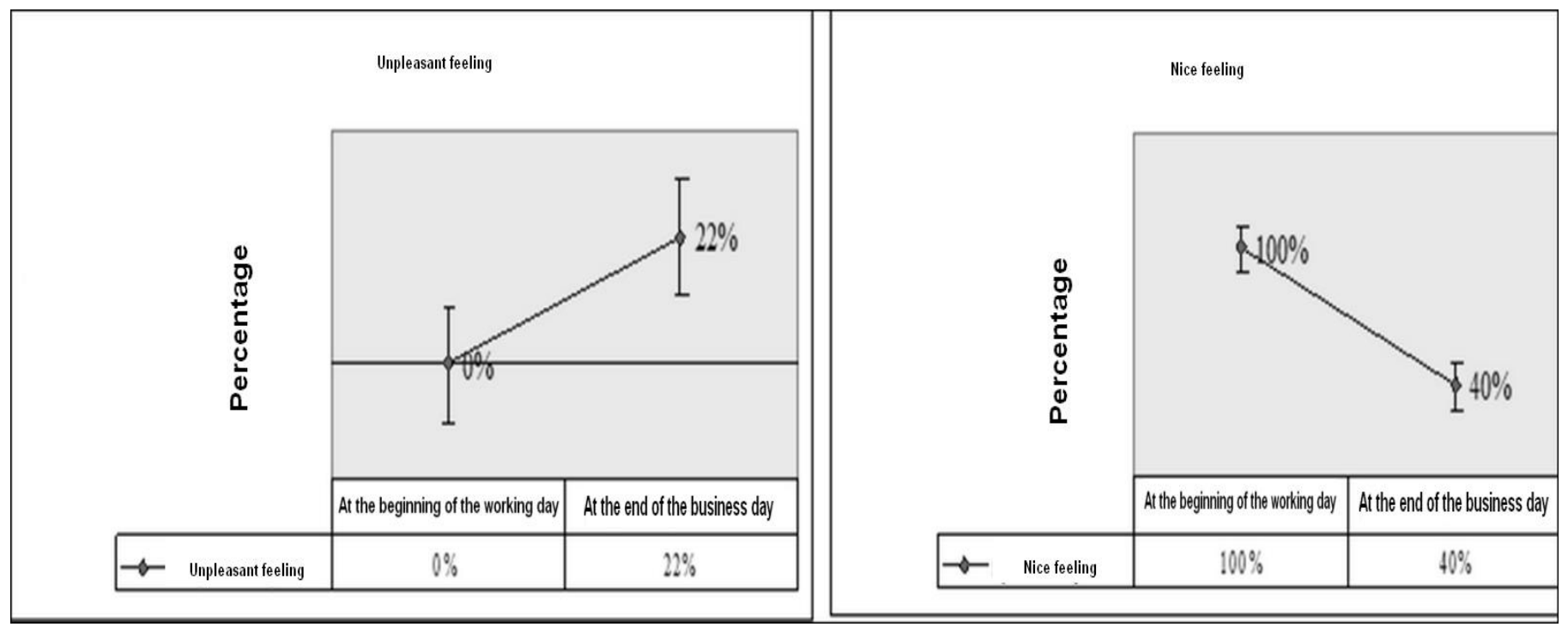

Diagram 7. Result in the globally friendly uncertain significance

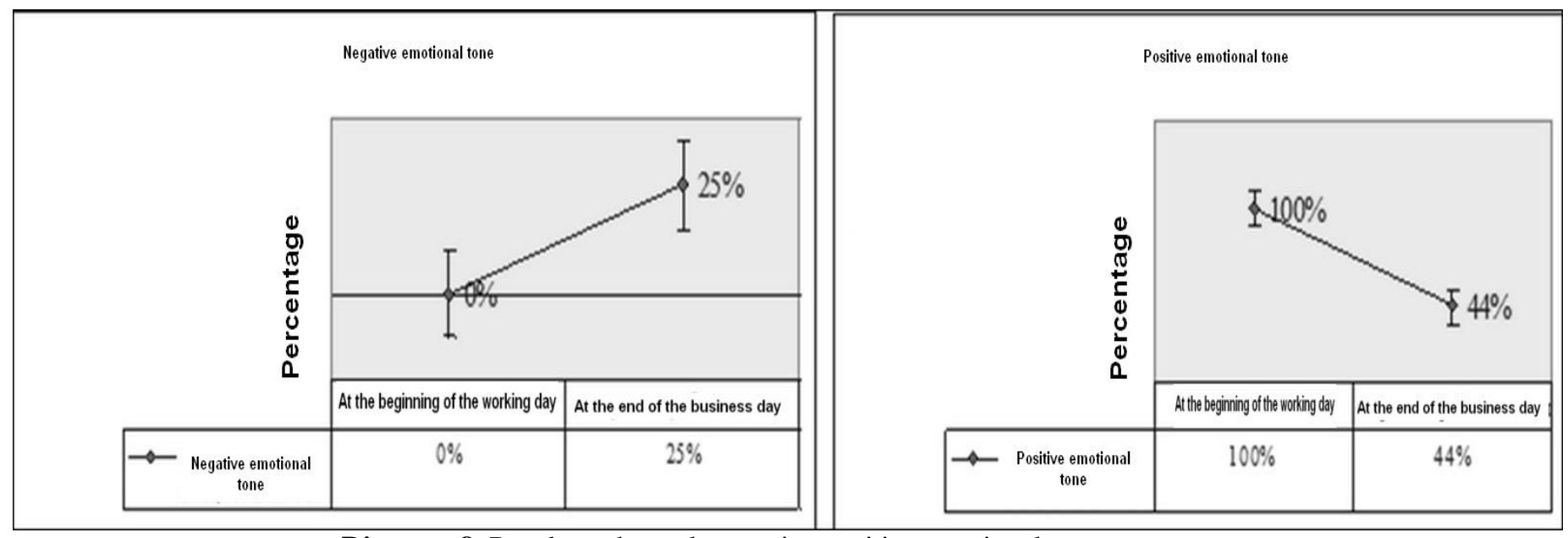

Diagram 8. Result on the scale negative positive emotional tone

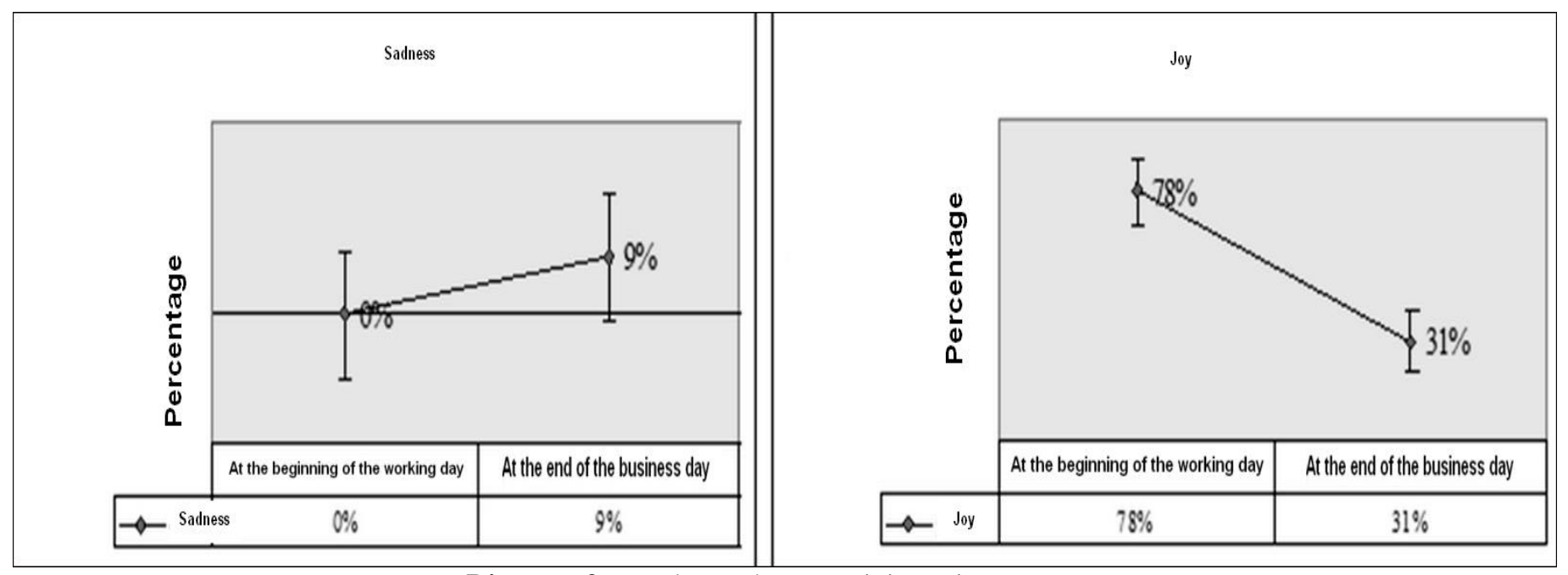

Diagram 9. Results on the sweet joint ratio 



\section{CONCLUSIONS}

The analysis of the results allows the individual differences in affective states to be distinguished from the similar trends in the affective states of the surveyed group of staff as a whole. The trend of a very strong emotional experience at the end of the working day, against the background of moderate indicators for the majority of the group, may be seen as a signal for a higher risk of the onset of a rapid-onset syndrome in a particular person.

For those working in the homes for medical and social care for children there is a tendency for a significant change in affective states during the working process. This change is related to the transition from positive to negative emotional experiences.

The timely assessment of the current affective status of the staff allows for the prevention of emotional burnout syndrome.
The test is a time-saving tool that can be applied in practice to other settings as it is a reliable means of preventing a burnout syndrome.

\section{REFERENCES}

1. Anisman, H., "Consequences of Stress", p.849-850, C: "Encyclopedia of Psychology", under the re. R. Korsini, Sofia: "Science and Art", 1998

2. Breeff, A., Prof. Stress Professional, p.895896, C: Encyclopedia of Psychology, under the name of Dr.

3. Isard, K., "Human emotions", Moscow: "Moscow University, 1980

4. Velichkov A, Radoslavova M, Methods for Psychodiagnostics, Sofia, 2005 